\title{
Implantação de Laboratório de Tecnologias como Espaço de Formação Profissional e Melhoria no Processo de Ensino-Aprendizagem de Ciência da Computação
}

\author{
Igor Medeiros Vanderlei - UFAPE - igor.vanderlei@ufape.edu.br \\ Rodrigo Gusmão de Carvalho Rocha - UFAPE - rodrigo.rocha@ufape.edu.br \\ Jean Carlos Teixeira de Araújo - UFAPE - jean.teixeira@ufape.edu.br \\ Mariel José Pimentel de Andrade - UFAPE - mariel.andrade@ufape.edu.br \\ Anderson Fernandes de Alencar - UFAPE - anderson.alencar@ufape.edu.br
}

\begin{abstract}
Resumo. Este artigo tem como objetivo discutir a implantação de um laboratório multidisciplinar voltado para o desenvolvimento de tecnologias sociais. Para verificar as contribuições deste laboratório no processo de formação dos alunos e para a sociedade em geral, foi realizada uma pesquisa de levantamento e documental. As análises dos dados indicaram que as atividades desenvolvidas no laboratório incentivaram os entrevistados a permanecerem no curso, auxiliaram no aprendizado de conteúdos específicos e na inserção dos egressos no mercado de trabalho. Além disso, a variedade de sistemas produzidos no laboratório permitiu contribuir com diversos setores da sociedade cumprindo também o seu papel social.
\end{abstract}

Palavras-chave: Ensino-aprendizagem, laboratório multidisciplinar, formação profissional.

\section{Implementation of a Technologies Laboratory as a Professional Training Space and Improvement in the Teaching-Learning Process of Computer Science}

\begin{abstract}
This paper aims to discuss the implementation of a multidisciplinary laboratory focused on the development of social technologies. To verify the contributions of this laboratory to the students' training process and to society, a survey and documentary research was carried out. The analysis of the data showed that the activities developed in the laboratory encouraged the interviewees to stay in the undergraduate program, helped in the learning of specific content, and the insertion of the graduates in the job market. Also, the variety of systems produced in the laboratory made it possible to contribute to various sectors of society while also fulfilling its social role.
\end{abstract}

Keywords: Teaching-learning, multidisciplinary laboratory, professional training.

\section{Introdução}

Cursos da grande área de Computação possuem uma presença marcante da abstração em algumas de suas disciplinas. Essa característica pode ampliar a dificuldade de aprendizagem e, como eventual consequência, uma redução na motivação de alguns alunos. Isso também pode culminar nos altos índices de reprovação e evasão, principalmente porque muitos desses alunos saem do Ensino Médio e chegam ao Ensino Superior sem essa habilidade desenvolvida. O que se observa é que, comumente, o aluno ingressante em cursos superiores da área de Computação e Informática e que não possui ou não consegue desenvolver a competência de abstração de problemas, não 
consegue um bom desempenho em disciplinas iniciais do curso, tais como algoritmos, cálculo, álgebra, entre outras (Piva e Freitas, 2010).

Almeida et al. (2002) observam também a ocorrência desse fenômeno e suas consequências nas disciplinas de programação. Pio, Castro e Castro Júnior (2006) explicam que grande parte dos professores e alunos de programação dedicam um esforço muito grande à construção de habilidades ligadas ao desenvolvimento algorítmico, e que o aluno só terá em mãos algo realmente próximo a um produto real após a passagem por várias disciplinas (como fundamentos da programação, algoritmo e estrutura de dados, linguagem de programação), nas quais muito pouco ou nada do que é desenvolvido faz parte do mundo físico em que vivemos. Essa dinâmica não contribui para reduzir o déficit na habilidade de abstração. É possível observar essa dificuldade, sobretudo, nas disciplinas iniciais. Assim, faz-se necessário buscar alternativas para contornar esse problema. Algumas iniciativas têm focado em repensar a prática metodológica do ensino de programação como, por exemplo, o uso paradigma de programação funcional (Castro, 2002), ambientes de programação visual (Ribeiro et al. 2012), aplicação de robótica (Martins et al., 2014) ou de jogos (Monclar et al., 2018).

Castro (2002) afirma que "um grande número de teorias de aprendizagem hoje utilizadas são categóricas ao afirmar que a aprendizagem só ocorre com a experiência, é o aprender fazendo". Embora esta afirmação tenha sido feita pelo autor no contexto da disciplina de fundamentos (ou introdução) à programação, podemos expandir sua ideia para o desenvolvimento de software. O processo de desenvolvimento de um software real envolve conhecimentos e habilidades desenvolvidas em várias disciplinas, tais como engenharia de software, linguagem de programação, banco de dados, gestão de projetos, entre outras. Assim, é no desenvolvimento de um projeto "real" que o aluno tem a oportunidade de superar a fragmentação do conhecimento imposta pela organização curricular tradicional (Silva, 2007).

Neste contexto, com o objetivo de proporcionar aos estudantes a vivência na prática de desenvolvimento de software, docentes da Universidade Federal do Agreste de Pernambuco conceberam o Laboratório Multidisciplinar de Tecnologias Sociais (LMTS). Este artigo descreve de forma geral o propósito do laboratório, sua estrutura e forma de funcionamento, além de demonstrar o impacto que ele causou e vem causando no curso de Computação, na instituição, na sociedade e na vida dos estudantes.

Este artigo está organizado da seguinte maneira: na Seção 2, são descritas a importância da realização de atividades práticas no Ensino Superior; em seguida, na Seção 3, o LMTS é apresentado de forma detalhada, descrevendo seu funcionamento, propósitos e conceitos; a Seção 4, por sua vez, traz os resultados conquistados através da implantação do laboratório, bem como a análise e discussão destes; por fim, a Seção 5 evidencia as considerações finais.

\section{Atividades Práticas no Ensino Superior}

Uma das principais funções das universidades é a formação de profissionais capacitados para atender à demanda da sociedade em uma determinada área. Para tanto, realizam-se estudos teóricos e atividades práticas, a fim de capacitar os estudantes para atuarem com excelência nos seus futuros ofícios. Tojal (1995) define a teoria como um "conjunto de conhecimentos sistematizados que se propõe explicar a ocorrência de determinados fenômenos" e a prática como o "conhecimento aplicado resultante de um saber advindo de um conjunto de conhecimentos sistematizados sobre determinados fenômenos". 
Reiteradamente, encontram-se depoimentos de alunos recém-formados das mais diversas áreas ou profissionais responsáveis pelas contratações afirmando que os cursos superiores não têm atingido os objetivos de formação profissional no que diz respeito às atividades práticas. Neste sentido, é comum que haja uma rápida mudança quando esses ex-alunos iniciam suas atividades profissionais, pois se deparam com uma grande lacuna entre o aprendizado adquirido e a realidade do ambiente de trabalho (Mattosinho et al., 2010). Em pesquisa realizada com profissionais recém-formados, foi constatado que metade dos entrevistados acreditam que não estão preparados para ingressarem no mercado de trabalho ao concluírem seus estudos nas faculdades/universidades, justamente por falta de experiência (Ipolito, 2017). Em outra pesquisa, desta vez realizada com recrutadores, chegou-se a uma conclusão similar, que os profissionais que estão se inserindo no mercado não estão tecnicamente preparados para atender às necessidades das empresas (Schuster, 2008). Ainda segundo a mesma pesquisa, as empresas buscam profissionais que possuam experiência prática de trabalho, independente da forma como estas experiências foram adquiridas, porém valorizando mais os conhecimentos adquiridos com a prática, com o dia a dia em outras empresas de Tecnologia (Schuster, 2008).

Em vista a reduzir o impacto da passagem da academia para o mercado de trabalho, o Conselho Nacional de Educação (CNE), órgão vinculado ao Ministério da Educação (MEC), através das diretrizes curriculares, preconiza que os projetos pedagógicos dos cursos da área de Computação devem incluir formas de integração entre teoria e prática (Brasil, 2016). Desta forma, espera-se que os discentes desenvolvam habilidades e competências para compreender os conceitos, os princípios e as teorias relacionadas à Ciência da Computação para o desenvolvimento de software e hardware e suas aplicações; além de conceber, aplicar e validar princípios, padrões e boas práticas no desenvolvimento de software; bem como analisar e criar modelos relacionados ao desenvolvimento de software (Brasil, 2016).

De forma similar, a Sociedade Brasileira de Computação (SBC) apresenta, nos Referenciais de Formação para Cursos de Graduação em Computação, o eixo de formação "Desenvolvimento de Sistemas", que deve contemplar o levantamento de requisitos funcionais e não-funcionais, sua análise, modelagem, projeto, implementação e teste (Zorzo et al., 2017). Neste sentido, os estudantes devem criar competências para desenvolver sistemas computacionais que atendam qualidade de processo e de produto, considerando princípios e boas práticas de engenharia de sistemas e engenharia de software, incluindo: Identificar, analisar, especificar e validar requisitos (Zorzo et al., 2017). Além disso, também devem projetar, implementar, testar e manter soluções computacionais em harmonia com o ambiente social e físico no seu entorno de aplicação, utilizando ambientes de desenvolvimento apropriados (Zorzo et al., 2017).

Em relação às práticas de ensino, a SBC recomenda empregar metodologias ativas, de forma que o aluno passe mais tempo em atividades nas quais seja protagonista no processo de ensino e aprendizagem (Zorzo et al., 2017). Além disso, também recomenda adotar metodologias como a Aprendizagem Baseada em Projetos e Aprendizagem Baseada em Problemas, entre tantas outras que estimulem a problematização e experiência do aluno (Zorzo et al., 2017), bem como procurar por interação com empresas, por meio, por exemplo, de estágio, laboratórios-empresa e empresa júnior. 


\section{Laboratório Multidisciplinar de Tecnologias Sociais (LMTS)}

O Laboratório Multidisciplinar de Tecnologias Sociais (LMTS) é uma iniciativa de professores e técnicos da Universidade Federal do Agreste de Pernambuco, com foco no desenvolvimento de tecnologias sociais. Este artigo adota o conceito de tecnologias sociais como sendo um conjunto de técnicas e metodologias transformadoras, desenvolvidas e/ou aplicadas na interação com a população e apropriadas por ela, que representam soluções para inclusão social e melhoria das condições de vida (ITS 2004).

O LMTS se trata de um espaço permanente de ensino, pesquisa, inovação tecnológica, extensão e de colaboração com a gestão institucional, contando com a participação de professores, técnicos e estudantes. Esse laboratório agrega inteligência coletiva e as múltiplas iniciativas, em curso ou idealizadas, em prol, especificamente, do desenvolvimento de software livres ou públicos para atender às demandas da universidade e da sociedade em geral. Através desse laboratório, vem sendo possível colocar em prática experimentos relacionados ao desenvolvimento de sistemas computacionais nas linhas de atuação propostas no projeto.

Para tornar a gestão e acompanhamento das atividades possíveis, foi necessário definir uma estrutura que permitisse que as decisões fossem tomadas democraticamente e com maior respaldo. Dessa forma, o laboratório se organiza com uma coordenação geral, vice-coordenação, que hoje acumula também a coordenação geral de desenvolvimento de software, além de um conselho gestor e de um colegiado pleno. Os discentes exercem suas atividades com diferentes tipos de vínculo, seja como bolsista ou voluntários de projetos de ensino, pesquisa, extensão e inovação tecnológica, como monitores, em estágios não obrigatórios, estágios obrigatórios supervisionados (ESO), como orientandos dos trabalhos de conclusão de curso e até como consultores (aqueles que interagem em contato mais técnico e esporádico).

Diversas atividades de ensino acontecem no LMTS, como a oferta de cursos e minicursos de formação para os discentes de Ciência da Computação, de outros cursos de graduação da instituição ou até mesmo para interessados externos à universidade promotora. São atividades de curta duração que objetivam atualizar e contribuir com a formação dos participantes. Outra atividade ligada ao ensino comumente realizada é a interação entre as disciplinas do Curso de Ciência da Computação com as atividades do laboratório, como a visita de professores e turmas para visualizarem na prática alguns conceitos aprendidos em sala-de-aula ou a apresentação de trabalhos em eventos por parte dos estudantes.

No quesito extensão, embora se possa considerar os cursos de curta duração acima citados como atividades de ensino, estes acabam também por serem entendidos como práticas extensionistas, visto que são abertos à sociedade em geral. Além destas, outras atividades são realizadas ou apoiadas, como por exemplo: desenvolvimento de aplicações para demandas da região; concepção de portais Web para os demais cursos e setores da universidade; e projetos de extensão ligados à informática nas escolas municipais da cidade.

Na dimensão de pesquisa, o LMTS também vem sendo consolidado através de projetos de pesquisa (iniciação científica) e inovação tecnológica, o que contribuiu para a concepção e para o desenvolvimento e consolidação deste laboratório. Atualmente, estão sendo desenvolvidos 5 projetos de pesquisa neste ambiente, que são provenientes de editais de PIBIC/PIC ou PIBITI da própria instituição. Alguns artigos foram escritos e publicados recentemente, sendo a maior parte na área de educação, e outros se encontram em fase de produção. 


\subsection{Métodos e conceitos envolvidos no processo de desenvolvimento de tecnologias do LMTS}

Seguindo os conceitos da Engenharia de Software, os projetos são conduzidos por um conjunto de etapas e métodos bem definidos, como também através do uso de ferramentas e tecnologias consolidadas. É utilizado como guia de referência o SWEBOK (Bourque e Fairley, 2014) e práticas do PMBOK (PMI, 2017), realizando apenas as devidas adaptações às necessidades do contexto.

Derivado de um projeto de pesquisa, o processo de desenvolvimento de software utilizado no LMTS começou a ser desenvolvido em 2018 e implementado em 2019, contemplando atividades essencialmente presenciais entre os times, mas que também foram adaptadas para a modalidade remota em razão da pandemia do COVID-19. O referido processo ainda está em fase de experimentação e adaptação, com variações ainda acontecendo por particularidades de cada projeto, principalmente pelas especificidades de ser realizado em um ambiente acadêmico. $O$ modelo atual de desenvolvimento de software está representado na Figura 1.

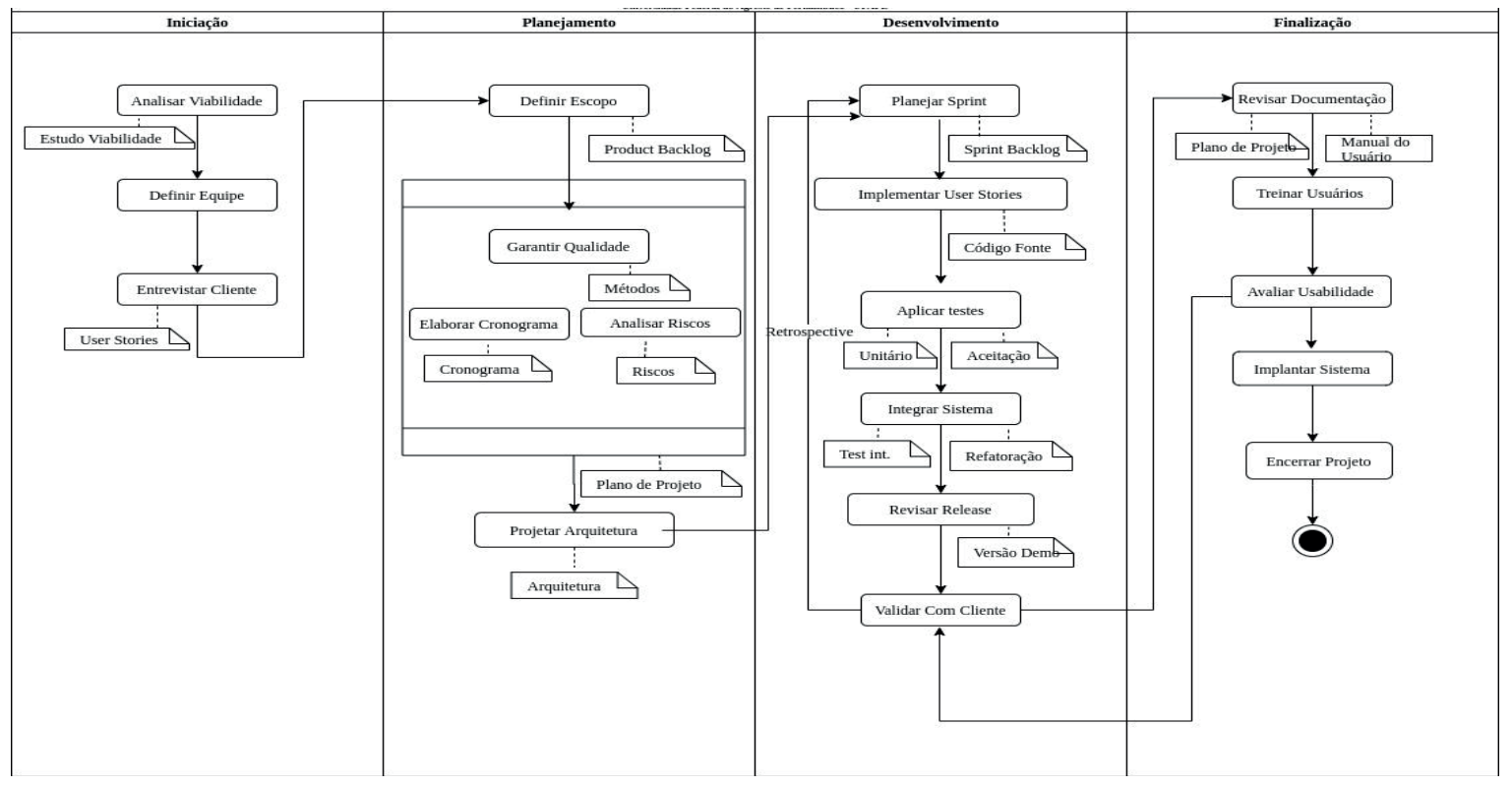

Figura 1. Modelo de desenvolvimento de software adotado pelo LMTS

Como pode ser observado, foi definido o fluxo das atividades mínimas e básicas para o formato de funcionamento do laboratório, desde o momento que recebe uma demanda de sistema, até o momento de implantação e treinamento. É importante ressaltar que essa é apenas a versão resumida em adaptação e em experimentação. Para gerenciar, acompanhar e executar as atividades contidas no processo, foram adotados os conceitos dos métodos ágeis (Campanelli e Parreiras, 2015), auxiliando na resolução de vários desafios. No sentido de gestão e acompanhamento dos projetos, utilizamos o SCRUM (Paasivaara et al., 2013) como referência.

Em todas as fases, as atividades são organizadas em ciclos de uma semana, chamados sprints, que têm início com uma reunião de definição das metas e planejamento das atividades, a sprint planning meeting. O resultado do planejamento é registrado no Redmine ${ }^{1}$, um software livre para gestão de projetos. Nos projetos mais

\footnotetext{
${ }^{1}$ Disponível em https://www.redmine.org/.
} 
recentes, temos testado a ferramenta Planning Poker ${ }^{2}$ para auxiliar de forma lúdica na análise do esforço necessário para concluir as tarefas.

Após o planejamento, a equipe de programação inicia imediatamente o desenvolvimento, utilizando diversas ferramentas. Como padrão, as tecnologias envolvidas seguem as linhas de desenvolvimento para Web e aplicativos móveis. Basicamente, adotamos as tecnologias: PHP com o framework Laravel ${ }^{3}$, HTML e Javascript na plataforma Web; para persistência, o PostgreSQL ${ }^{4}$; e Android Studio ${ }^{5}$ para os sistemas móveis. Como o LMTS tem propósito de gerar tecnologias sociais, todos os projetos estão disponíveis no GitHub ${ }^{6}$. Além disso, temos bons resultados com a utilização do Slack ${ }^{7}$ para manter a comunicação entre estudantes e professores ao longo de toda semana. Ao fim da sprint, os resultados são apresentados em uma reunião e inicia-se um novo ciclo.

Esta organização atual, que foi definida e adaptada ao longo da existência do laboratório, tem se mostrado adequada ao trabalho no contexto acadêmico. Vale salientar que, mesmo com a imposição do isolamento social causado pela pandemia da COVID-19, as atividades do laboratório continuaram sendo executadas de forma remota, com pequenas mudanças na rotina de trabalho.

\section{Resultados e Discussões}

Desde sua concepção, o LMTS tem obtido de forma contínua resultados positivos em vários aspectos: para os professores, para os cursos envolvidos, para a sociedade, para a universidade, para o próprio laboratório e, principalmente, para os estudantes. Neste período foram desenvolvidos 19 projetos, sendo 15 sistemas para Web, 2 aplicativos mobile e 2 APIs. Esses sistemas, que estão apresentados de forma resumida no Quadro 1, foram registrados ou estão em processo de registro através do Instituto Nacional da Propriedade Industrial (INPI). Outras atividades, como a elaboração de sites dos cursos, a realização de eventos, oficinas, minicursos e projetos de pesquisa, extensão ou inovação tecnológica sem relação com desenvolvimento de software também foram realizadas, mas não foram contempladas neste documento porque estão fora do escopo desta análise.

O desenvolvimento desse número significativo de projetos foi possível graças à participação de 25 estudantes do curso de Ciência da Computação e 12 professores de diversos cursos da instituição. A Figura 2 apresenta a linha do tempo e dados gerais dos projetos desenvolvidos. Esses dados foram coletados a partir da análise das informações contidas nos repositórios do GitHub do laboratório. Como pode ser observado, no início do laboratório apenas um projeto (o Notum) foi desenvolvido. Nesta época, havia apenas um aluno bolsista de inovação tecnológica e três professores envolvidos. Com o passar do tempo, novos estudantes e professores passaram a integrar o laboratório e, desta forma, foi possível desenvolver vários projetos paralelamente. Outro fator importante que pode ser observado a partir da Figura 2 é o fato de que os projetos mais recentes, a partir do segundo semestre de 2019, tiveram o período de desenvolvimento mais rápido, devido à maturidade do processo de desenvolvimento de software adotado.

\footnotetext{
${ }^{2}$ Disponível em https://www.planningpoker.com/.

${ }^{3}$ Disponível em https://laravel.com/.

${ }^{4}$ Disponível em https://www.postgresql.org/.

${ }^{5}$ Disponível em https://developer.android.com/studio.

${ }^{6}$ Disponível em https://github.com/.

${ }^{7}$ Disponível em https://slack.com/.
} 


\begin{tabular}{|c|c|}
\hline Projeto & Finalidade \\
\hline API Ufape & $\begin{array}{l}\text { Prover uma base unificada de informações (departamentos, logins e etc) para os sistemas } \\
\text { institucionais. }\end{array}$ \\
\hline App Ufape & Aplicativo mobile que fornece acesso à informações sobre a universidade. \\
\hline Avaliar & Acompanhamento da aprendizagem de estudantes do ensino infantil e básico. \\
\hline Colinas Job & Implementar um banco de currículos e oportunidades de empregos. \\
\hline ConectAEE & $\begin{array}{l}\text { Estabelecer a comunicação Escola/Terapeutas/Família no âmbito do Atendimento Educacional } \\
\text { Especializado }\end{array}$ \\
\hline Encontre e Compre & $\begin{array}{l}\text { Fornecer de informações sobre estabelecimentos comerciais que estão atendendo por delivery } \\
\text { durante a pandemia da Covid } 19 \text {. }\end{array}$ \\
\hline Gestāo de Eventos & $\begin{array}{l}\text { Facilitar o gerenciamento de eventos científicos (inscrição, pagamento, programação, submissão } \\
\text { de trabalhos e etc) }\end{array}$ \\
\hline Gestāo PPC & Facilitar o processo de criação e atualização dos projetos pedagógicos dos cursos. \\
\hline Glossário Paulo Freire & Facilitar 0 acesso aos recursos audiovisuais disponíveis com/sobre o educador Paulo Freire. \\
\hline Ingresso Extravestibular & $\begin{array}{l}\text { Gerenciar editais, receber as inscrições e tramitar os processos de ingresso extra vestibular na } \\
\text { universidade }\end{array}$ \\
\hline Notum & Automatizar o processo de emissão de certificados da universidade. \\
\hline Psicultec & Planejar e controlar os processos produtivos em uma psicultura de tilápias. \\
\hline SofiaEdu & $\begin{array}{l}\text { Repositório para compartilhamento de atividades educativas do ensino infantil, que utilizam } \\
\text { tecnologia. }\end{array}$ \\
\hline Solicita & Informatizar o processo de solicitação de documentos na universidade. \\
\hline SoS Enade & Confecção e realização de simulados para preparação dos estudantes para o ENADE. \\
\hline Submeta & $\begin{array}{l}\text { Informatizar os processos de submissão e avaliação de projetos em editais de ensino, pesquisa e } \\
\text { extensão. }\end{array}$ \\
\hline Vacina Garanhuns (API) & $\begin{array}{l}\text { Unificar a base de informações sobre as solicitações de vacina realizadas no aplicativo mobile } \\
\text { Vacina Garanhuns. }\end{array}$ \\
\hline Vacina Garanhus & $\begin{array}{l}\text { Disponibilizar o agendamento de vacinas, junto à secretaria municipal de saúde, para pacientes } \\
\text { com dificuldade de locomoção ao posto de saúde. }\end{array}$ \\
\hline Vô na Feira & Organização de feiras para venda de produtos agroecológicos e da agricultura familiar. \\
\hline
\end{tabular}

\section{Quadro 1. Sistemas desenvolvidos no LMTS}

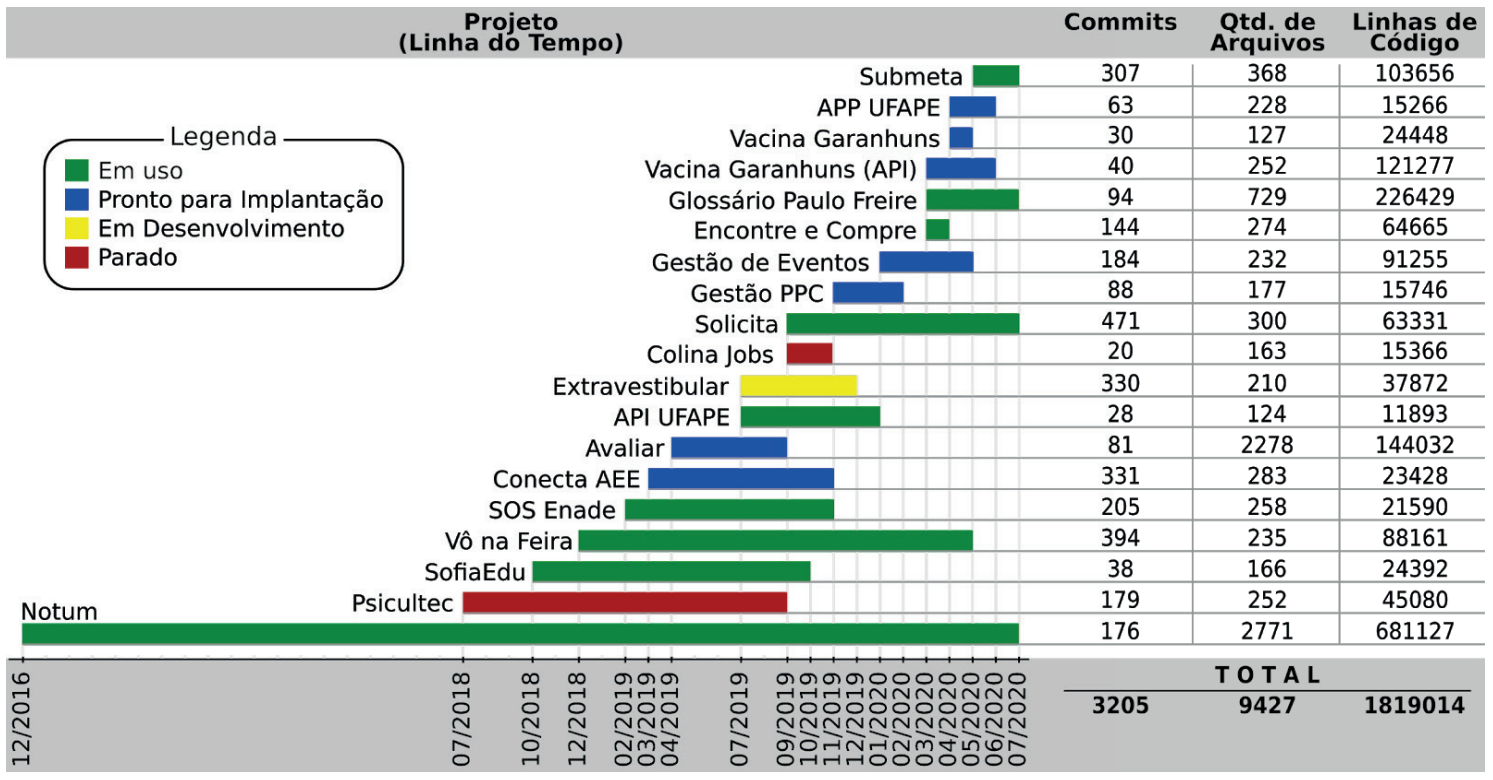

Figura 2. Linha do tempo e dados gerais do desenvolvimento dos projetos

Cabe observar que as marcações temporais de início e fim dos projetos são baseados no primeiro e último commit realizados no GitHub. Desta forma, um projeto que já foi concluído, está em produção e teve uma pequena atualização posterior à sua conclusão, está representado por um tempo maior. Em outras palavras, esta figura apresenta o período em que o projeto teve alguma atividade, não sendo necessariamente com desenvolvimento intenso. Maiores informações sobre os projetos podem ser obtidas na página de portfólio do laboratório, disponível em: http://lmts.uag.ufrpe.br/br/content/portif\%C3\%B3lio. 
Os professores e os cursos envolvidos tiveram benefícios claros, como o registro dos software, a oportunidade de executar pesquisa em um ambiente de produção, a realização de projetos com professores de outras áreas, com troca de conhecimentos e experiências, um ambiente propício para a realização de visitas técnicas e práticas de disciplinas, entre outros. A Universidade adotou alguns dos sistemas desenvolvidos para uso institucional, como o "Notum", o "Solicita" e o "Submeta". Por sua vez, o público externo à universidade também se beneficiou com a utilização dos softwares produzidos para atender à demandas locais, como os sistemas já em utilização "Vô na Feira", "SOfiaEdu Planos de Aula" e "Encontre \& Compre". Por fim, para investigar a percepção dos discentes em relação às experiências proporcionadas pelo laboratório, foi elaborado um questionário, cujo resultado e análises se encontram na seção a seguir.

\subsection{Percepção dos discentes sobre o LMTS}

É possível afirmar que o LMTS já é considerado pelos alunos do Curso como uma referência para uma atuação mais profissional, para ganho de experiência e aprendizagem. Para confirmar isso, foi aplicado um questionário com 21 membros e ex-membros do laboratório com o intuito de identificar qual a percepção dos discentes em relação ao LMTS e a fim de compreender melhor o seu impacto. De acordo com o levantamento, 28,6\% das respostas corresponde a egressos do curso de Ciência da Computação, enquanto os $71,4 \%$ restantes foram de discentes que ainda estão vinculados ao curso, dos quais $14,3 \%$ estão na primeira metade (do primeiro ao quarto período), enquanto $57,1 \%$ restante estão na segunda metade do curso (do quinto ao nono período). Além disso, dos participantes do questionário, 61,9\% são ex-membros do LMTS, enquanto 38,1\% ainda estão vinculados ao laboratório.

Ao serem questionados se as atividades desenvolvidas no LMTS influenciaram ou facilitaram o aprendizado em disciplinas do curso, 95,2\% dos entrevistados afirmaram que sim, enquanto $4,8 \%$ informaram que não. Sobre a opinião deles em relação aos benefícios advindos da existência de laboratórios no formato do LMTS, a resposta foi unânime, ou seja, $100 \%$ dos entrevistados responderam que esse tipo de laboratório incentiva os alunos a permanecerem no curso. Outrossim, todos os entrevistados também informaram que a experiência no laboratório contribuiu com o aprendizado de novas tecnologias, com a aquisição de experiência profissional, e com o desenvolvimento de conhecimento prático em processos e metodologias de desenvolvimento de software. Tudo isso demonstra a importância do LMTS nos aspectos relacionados ao ensino, cumprindo também o seu papel social.

Em relação à situação empregatícia dos discentes, 47,6\% ainda não possuem nenhum vínculo empregatício e também não prestam nenhum tipo de serviço de TI, enquanto $52,4 \%$ já estão empregados. Desses com vínculo empregatício, todos eles informaram que o conhecimento obtido através do LMTS possibilitou um menor tempo de adaptação no trabalho. Esses dados corroboram a dificuldade da adaptação e da inserção dos egressos dos cursos de tecnologia por questões de experiência e de conhecimento prático. Neste caso, não houve problemas na inserção dos egressos no ambiente de trabalho, em razão da experiência obtida a partir do laboratório. Esse é um importante resultado do laboratório, pois permite uma preparação técnica e profissional mais real dos seus estudantes, facilitando o ingresso no mercado de trabalho.

Vale salientar que diversos alunos, antes mesmo de concluírem o curso, foram contratados por empresas de tecnologia, atuando com desenvolvimento de software. Por esse motivo, foi questionado o quão importante foram as atividades desenvolvidas no LMTS para a conquista desse emprego. Dos entrevistados, $81,8 \%$ atribuíram a nota 
máxima, ou seja, que o LMTS foi muito importante para a conquista do emprego. É importante destacar que estes alunos ressaltam que em suas entrevistas de seleção de emprego, o fato de ter a experiência prática em um laboratório que oferece um ambiente profissional, com projetos e clientes reais, influenciou diretamente nas contratações. Inclusive, para aquelas empresas com critérios de seleção mais rígidos, geralmente o portfólio dos candidatos é avaliado através do próprio GitHub.

\section{Considerações Finais}

A formação inicial (acadêmica e profissional) do cientista da computação é um desafio, não só pela natureza da própria área do conhecimento em si, que está em permanente atualização e reinvenção, mas também devido às relações de mercado que se complexificam cotidianamente. Pensando nisso, o LMTS foi criado como um espaço de ensino, pesquisa, extensão e inovação tecnológica, voltado para o desenvolvimento de software livres ou públicos, objetivando atender as demandas da universidade e da sociedade em geral. A partir da análise dos dados levantados através de um questionário com membros e ex-membros do laboratório, foi percebido diversas contribuições, tanto nos aspectos de ensino quanto na inserção no mercado de trabalho. Além disso, também é importante destacar que algumas disciplinas do curso de computação influenciam diretamente na qualidade da mão de obra dos discentes envolvidos no LMTS. Sendo que, para determinados projetos, algumas dessas disciplinas são consideradas como pré-requisito para participação do discente no projeto.

Além dos resultados positivos, é possível identificar alguns desafios inerentes à proposta de um laboratório nos moldes do LMTS, tais como: conhecimento técnico não suficiente para alguns projetos, levando a um maior tempo de desenvolvimento; os desenvolvedores são alunos e, apesar de fazerem parte de um ambiente profissional e produtivo, é necessário considerar que os alunos possuem horários e restrições particulares (transporte, semana de provas, etc.). Apesar dessas particularidades, é possível afirmar que o LMTS é um espaço de aproximação permanente entre as demandas acadêmicas, sociais e de mercado, reduzindo o distanciamento entre o que vem sendo concebido com a teoria em contraposição à prática.

\section{Referências Bibliográficas}

ALMEIDA, E. S.; COSTA, E. B.; BRAGA, J. D. H.; SILVA, K. S.; PAES, R. B. e ALMEIDA, A. A. M. AMBAP: Um Ambiente de Apoio ao Aprendizado de Programação. In X WORKSHOP SOBRE EDUCAÇÃO EM COMPUTAÇÃO. 2002. Florianópolis/SC. Anais do WEI 2002.

BOURQUE, P. e FAIRLEY, R. E. Guide to the Software Engineering Body of Knowledge", Version 3.0. 2014. IEEE Computer Society. Disponível em www.swebok.org.

CAMPANELLI, A. S. e PARREIRAS, F. S. Agile methods tailoring-a systematic literature review. Journal of Systems and Software. 2015. Elsevier, v. 110, p. $85-100$.

CASTRO, T. H. C.; CASTRO Jr, A. N.; MENEZES, C. S.; BOERES, M. C. S. e RAUBER, M. C. P. V. Utilizando Programação Funcional em Disciplinas Introdutórias de Computação. In: X WORKSHOP SOBRE EDUCAÇÃO EM COMPUTAÇÃO. 2002. Florianópolis/SC.

BRASIL. Resolução CNE/CES no 05/2016. Diretrizes Curriculares Nacionais para os cursos de graduação na área da Computação. 2016. 
IPOLITO, S. P. Empregabilidade dos recém-formados em administração no município de Cacoal/RO. Fundação Universidade Federal de Rondônia. Campus Professor Francisco Gonçalves Quiles. 2017. Cacoal/RO.

ITS BRASIL. Caderno de Debate - Tecnologia Social no Brasil. São Paulo: ITS. 2004: 26.

MARTINS, J. P. A.; ROCHA, C. A.; RAMOS, B. A. e VANDERLEI, I. M. LogobotUm sistema robótico simulador da linguagem logo para auxílio no aprendizado de programação. In ANAIS DO XXV SIMPÓSIO BRASILEIRO DE INFORMÁTICA NA EDUCAÇÃO. 2014.

MATTOSINHO, M. M. S.; COELHO, M. S.; MEIRELlES, B. H. S.; SOUZA S. S. e ARGENTA C. E. Mundo do trabalho: alguns aspectos vivenciados pelos profissionais recém-formados em enfermagem. Acta paul. enferm. 2010. Vol. 23(4):466-72.

MONCLAR, R. S.; SILVA, M. A. e XEXÉO, G. Jogos com Propósito para o Ensino de Programação. In Proc. of XVII SBGAMES. 2018. Foz do Iguaçu-PR.

PAASIVAARA, M.; LASSENIUS, C.; DAMIAN, D.; RÄTY, P. e SCHRÖTER, A. Teaching students global software engineering skills using distributed scrum. In Proc. of The International Conference On Software Engineering (ICSE'13). 2013. IEEE, 1128-1137.

PIO, J. L. S.; CASTRO, T. H. C. e JÚNIOR A. N. C. A Robótica Móvel como Instrumento de Apoio à Aprendizagem de Computação. In: Anais do XVII Simpósio Brasileiro de Informática na Educação - SBIE. 2006.

PIVA JR., D. e FREITAS, R. L. Estratégias para melhorar os processos de abstração na disciplina de Algoritmos. In: $\mathbf{2 1}^{\circ}$ Simpósio Brasileiro de Informática na Educação (SBIE). 2010. João Pessoa, Brasil.

PMI. A Guide to the Project Management Body of Knowledge (PMBOK® Guide)-Sixth Edition. Project Management Institute PMI. 2017.

RIBEIRO, R. D. S.; BRANDÃO, L. D. O. e BRANDÃO, A. A. Uma visão do cenário Nacional do Ensino de Algoritmos e Programação: uma proposta baseada no Paradigma de Programação Visual. In Anais do Simpósio Brasileiro de Informática na Educação. 2012. (Vol. 23, No. 1).

SILVA, T. T. Documentos de identidade - Uma Introdução às teorias do currículo. Autêntica. 2007. Belo Horizonte.

SCHUSTER, M. E. Mercado de trabalho de tecnologia da informação: O Perfil dos profissionais demandado. Universidade Federal do Rio Grande do Sul. Trabalho de Conclusão de Curso de Graduação. Porto Alegre/RS. 2008.

TOJAL, J. B. G. A dicotomia Teoria/Prática na Educação Física. Anais da III Semana de Educação Física. 1995. Universidade São Judas Tadeu - São Paulo, p.17-21.

ZORZO, A. F., NUNES, D., MATOS, E., STEINMACHER, I., LEITE, J., ARAÚJO, R. M., CORREIA, R., MARTINS, S. Referenciais de Formação para os Cursos de Graduação em Computação. Sociedade Brasileira de Computação (SBC). 2017. 153p. 